\title{
En bloc proximal peri-mesenteric clearance for pancreatic head cancer surgery
}

\author{
Mee Joo Kang and Sun-Whe Kim
}

\section{Center for Liver and Pancreatobiliary Cancer, National Cancer Center, Goyang, Korea}

\begin{abstract}
The superior mesenteric artery (SMA) first approach and meso-pancreas excision (MPE) during pancreatoduodenectomy (PD) for pancreatic head cancer have been suggested for complete local tumor control, less operative blood loss, and early determination of resectability. However, SMA-first approach is merely a mode of approach and the concept of MPE has been challenged due to its anatomical obscurity. Dissection around proximal mesenteric vessels, superior mesenteric vein and SMA, is a critical procedure point for local tumor control as tumor infiltration is frequently observed both at the time of initial diagnosis and recurrence. The meso-pancreas, which encompasses the soft tissue between the uncinated process and SMA, does not include all the aforementioned points of proximal mesenteric areas. Therefore, the authors propose a new terminology named, "en bloc proximal peri-mesenteric clearance (PPMC)", to describe the removal of all the lymph nodes including soft tissue around proximal mesenteric vessels, especially the SMA, to ensure complete local tumor control of pancreatic head cancer. The SMA-first approach applied either by the mesenteric approach or supra-colic approach can make this procedure more feasible. The extent of the circumferential dissection of the peri-SMA nerve plexus can be adjusted according to the primary disease. PPMC including the removal of all lymph nodes around the proximal SMA may be considered as a standard extent of PD for pancreatic head cancer. (Ann Hepatobiliary Pancreat Surg 2020;24:389-395)
\end{abstract}

Key Words: Pancreatic neoplasms; Pancreaticoduodenectomy; Proximal peri-mesenteric clearance; SMA-first approach; Mesopancreas excision

\section{INTRODUCTION}

During pancreatoduodenectomy (PD) for pancreatic head cancer, the dissection of the pancreatic head, especially the uncinate process, from mesenteric vessels is a critical step that has immense effects on early and late postoperative outcomes. A high rate of anatomical variation of superior mesenteric vessel branches and tributaries makes the dissection more difficult and prone to frequent intraoperative bleeding. ${ }^{1}$ Moreover, a relatively complicated anatomy in this area is a serious obstacle in achieving R0 resection using conventional approaches, especially when clearing lymph nodes (LNs) around the superior mesenteric artery (SMA)., ${ }^{2,3}$ A superior part of the mesenteric root is in a close proximity of tumors located at the head of the pancreas with a high risk of tumor invasion, R1 resection and local recurrence. ${ }^{4,5}$ Consequently, the concepts of artery-first approach ${ }^{6-8}$ and meso-pancreas excision $^{9-11}$ have been introduced to overcome technical challenges and improve the completeness of PD as an oncologic surgery.

Although the survival gain in the artery-first approach has been reported in recent matched-pair analysis ${ }^{12}$ and meta-analysis, ${ }^{3}$ it still does not have a sufficiently high level of evidence to consider this approach as one that improves survival. Furthermore, lymphadenectomy extended to the left side of the SMA has been suggested to improve the $\mathrm{R} 0$ resection rate and reduce local recurrences. ${ }^{10,13}$ However, the meso-pancreas excision did not include this extended scope of lymphadenectomy. ${ }^{9,14}$ Therefore, the authors aim to review the progress of these two concepts and propose a new procedure named: en bloc proximal peri-mesenteric clearance (PPMC), which uses both of these perspectives on surgical approach and oncological

Received: November 11, 2020; Accepted: November 14, 2020

Corresponding author: Sun-Whe Kim

Center for Liver and Pancreatobiliary Cancer, National Cancer Center, 323 Ilsan-ro, Ilsandong-gu, Goyang 10408, Korea Tel: +82-31-920-0891, Fax: +82-31-920-2799, E-mail: sunkim@ncc.re.kr

Copyright (C) 2020 by The Korean Association of Hepato-Biliary-Pancreatic Surgery

This is an Open Access article distributed under the terms of the Creative Commons Attribution Non-Commercial License (http://creativecommons.org/ censes/by-nc/4.0) which permits unrestricted non-commercial use, distribution, and reproduction in any medium, provided the original work is properly cited. Annals of Hepato-Biliary-Pancreatic Surgery • pISSN: 2508-5778 - elSSN: 2508-5859 
clearance of pancreatic head cancer.

\section{CONVENTIONAL APPROACH FOR DISSECTION OF THE PANCREATIC HEAD}

The conventional approach for pancreatic head dissection starts with separating the portal vein (PV) and superior mesenteric vein (SMV) from the pancreas by dividing small tributaries to the PV/SMV and retracting the freed PV/SMV to the anterior and left side to expose the uncinate process and peri-SMA retroperitoneal area. During this procedure, small pancreatic branches from the SMA, and pancreatic head nerve plexus are divided; the inferior pancreaticoduodenal artery (IPDA), which is a branch of the SMA that usually arises from it, or uncommonly from the first jejunal artery (Ja1), is also divided. One or two proximal jejunal veins ( Jv) that usually run dorsal to the SMA and are occasionally located very close to the tumor can be identified in the caudal area of dissection. ${ }^{15,16}$ Injuring these small arteries or tributaries might cause significant bleeding. ${ }^{1}$ The aim of this procedure is to remove all tissue including nerves and LNs on the right side of the SMA with or without tissue clearance posterior to the SMA. This is the last step before the specimen is taken out. This area is also known as a retroperitoneal resection margin. A retrospective review of recurrence patterns after the conventional approach for uncinate process cancer revealed that the local recurrence was more frequently identified in this area. ${ }^{17}$

\section{VARIOUS SMA-FIRST APPROACHES}

The artery-first approach mostly refers to the SMA-first approach in PD. In the SMA-first approach, tissue around the SMA is dissected firstly to separate the uncinate process from the SMA, before approaching the PV/SMV and transecting the neck of the pancreas.

Until now, six categories of the SMA-first approach for PD have been reported, which are posterior, ${ }^{18}$ medial uncinate, ${ }^{19,20}$ inferior infracolic (mesenteric), ${ }^{7,8}$ left posterior, ${ }^{5}$ inferior supracolic (anterior), ${ }^{21}$ and superior approaches. ${ }^{22}$ The rationale for the SMA-first approach includes early determination of resectability, reduced blood loss by early vascular control, and increased possibility of R0 resection by dissecting tissue around the SMA more efficiently from an oncological and technical standpoint compared to that of the traditional SMA-last approach. If peri-SMA nerve plexus tumor involvement can be confirmed by frozen section biopsy during the SMA-first approach, unnecessary further surgery can be avoided. This advantage is more significant with the evolving concept of borderline resectable diseases and the widespread application of neoadjuvant treatment as difficulties in the interpretation of periarterial stranding on imaging studies after neoadjuvant treatment demanded early intraoperative resectablity determination during PD. ${ }^{22}$ Additionally, the SMA-first approach makes it possible to dissect and control pancreatic arteries and Jvs under direct vision; hence, enabling early vascular control and possibly reducing intraoperative blood loss. The posterior approach appeared to have better postoperative outcomes in terms of intraoperative blood loss, postoperative stay, possibility of complications, pancreatic fistula, delayed gastric emptying, and intra-abdominal infections. ${ }^{3}$

Most importantly, however, a better surgical field obtained by the SMA-first approach enables a more complete dissection around the SMA, which is expected to reduce the possibility of $\mathrm{R} 1$ resection. Conventionally, positive retroperitoneal margin is associated with poor survival outcomes. ${ }^{23}$ From an oncological point of view, several concepts were added to the abovementioned surgical approaches, including no-touch isolation to the inferior infracolic approach, ${ }^{24}$ meso-pancreas excision to the left posterior approach, ${ }^{4}$ and tailored SMA dissection according to the extent of the tumor. ${ }^{25}$ However, the oncological benefits of the SMA-first approach are still controversial. A recent meta-analysis revealed an improved 3-year overall survival rate in SMA-first approach, especially with the posterior approach. ${ }^{3}$ However, a matched-pair analysis stratified into tumor resectability also revealed the benefit of the SMA-first approach on the R0 resection rate and overall survival in resectable pancreatic head cancer, yet no benefits were reported on borderline resectable pancreatic head cancer in this analysis. ${ }^{12}$ Similarly, a recent multicenter randomized controlled trial failed to prove the advantage of the SMA-first approach in achieving R0 resection. ${ }^{26}$ Three and five year overall survival rates also did not differ after the tailored dissection of the tissue around the SMA, ranging from limited to regional lym- 
phadenectomy to hemi- or total circumferential nerve plexus dissection. ${ }^{2}$

\section{MESO-PANCREAS AND MESO-PANCREATODUODENAL EXCISION}

Since the conception of the meso-pancreas, which includes mainly the right and posterior sides at the caudal level of SMA, ${ }^{9}$ meso-pancreas or meso-pancreatoduodenum excision has been proposed to improve the complete local tumor control of pancreatic head cancer. ${ }^{10,13,25,27}$ The mesopancreas has been suggested as the local recurrence site which is associated with long-term prognosis, ${ }^{10,28}$ and the SMA-first approach with a tailored level of SMA dissection has been suggested for complete meso-pancreas excision. $^{25,28}$ However, there have been debates on the concept of the meso-pancreas and the terminology and oncological benefits of dissecting it or the meso-pancreatoduodenum. $^{27}$ By definition, the meso-pancreas is an area with soft tissue supplying the pancreatic head, which contains vessels, nerves and lymphatic tissue running into or from the head of pancreas. Nevertheless, it is difficult to define its anatomical boundary, and some reported the area to be boundless and has continuity with the para-aortic space. ${ }^{13}$ Moreover, the majority of the evidence on the benefit of dissecting this area were based on retrospective studies with a small number of study subjects. ${ }^{27}$ Therefore, a consensus on the concept of meso-pancreas should be established, and higher levels of evidence with randomized clinical trials are needed to prove its oncological significance.

\section{PROGNOSTIC IMPLICATION OF THE LYMPH NODES ALONG THE PROXIMAL SMA}

According to the review of the local recurrence pattern after PD for pancreatic head cancer, nodal recurrence in the peri-SMA area was more frequent in the left side than the right or posterior side of the SMA (Fig. 1). ${ }^{17}$ This is expected as the right-sided soft tissue including LNs are usually removed during conventional PD.

The sub-station classification of LN No. 14 has changed from $14 \mathrm{a}, 14 \mathrm{~b}, 14 \mathrm{c}$, and $14 \mathrm{~d}$ to $14 \mathrm{p}$ and $14 \mathrm{~d}$ in the new

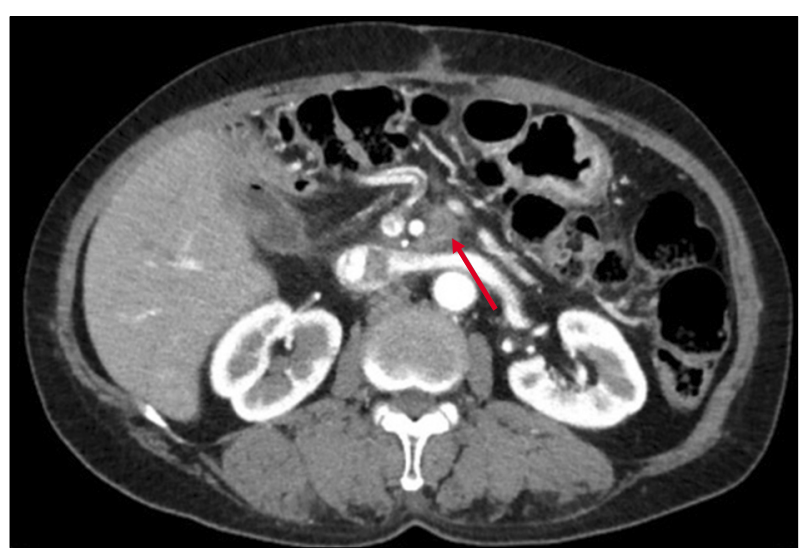

Fig. 1. Nodal type local recurrence of pancreatic head cancer on the left side of SMA.

Japanese LN station system. ${ }^{29}$ While the locations of LNs are numbered in cranio-caudal order, whether the LNs are located in the right or left side of SMA has not been taken into consideration. However, LNs located in the right or left side of the SMA have been recognized to have different clinical implications in practice. Standard PD described in literatures regarding the extent of surgery, included only the right side of the SMA in their extent of surgery. ${ }^{30,31}$ In contrast, LN station No. 14 located in the left side of the SMA has been considered to be associated with very poor prognosis, similar to the para-aortic (LN station No. 16) LN metastasis. ${ }^{10}$ Although LN metastasis to station No. 14 located in the left side of the SMA should be differentiated from metastasis to LN station No. 16 which is considered a systemic disease, there is no solid evidence on the prognostic impact of dissecting LN station No. 14 in this perspective, currently. In this context, total mesopancreatoduodenum excision (tMPDe) included lymphadenectomy in the left side of the SMA, though the long-term survival outcome is yet to be reported. ${ }^{10,28}$

In fact, any types of effort to increase $\mathrm{R} 0$ resection rate is worthwhile to achieve complete local tumor control. The conventional concept of R0 resection is based on the direct spread of the tumor along the primary organ, or perineural or adjacent soft tissue. Therefore, the current concept of R0 resection is not applicable to $\mathrm{LN}$ metastasis. However, tumor clearance regarding LN metastasis needs to be incorporated in the concept of oncological radicality. In this regard, the dissection of LN station No. 14 including those located in the left side of the SMA may be valuable for staging and also increasing oncological clearance. 
However, additional studies are required to confirm these oncological benefits.

\section{EN BLOC PROXIMAL PERI-MESENTERIC CLEARANCE}

LN station No. 14 located in the left side of the SMA can be distributed from the origin of the SMA to the level of origin of Ja1 or Ja2. They are located in the meso-jejunum mesentery, not in the meso-pancreas. Therefore, mesopancreas excision may not be an adequate terminology to define this procedure including the dissection of all the LNs around the proximal SMA as well as the SMV. In addition, the SMA-first approach should be understood as a type of technical approach, while meso-pancreas excision should be considered as an extent or range of re- section. Nevertheless, both concepts should be adopted to possibly achieve higher $\mathrm{R} 0$ resection rates.

As a result, the authors suggest a concept of "En bloc Proximal Peri-Mesenteric Clearance (PPMC)". This procedure can be defined as one including soft tissue clearance around the SMA from its origin to the level of Ja1 and Ja2 origin, with emphasis on the circumferential lymphadenectomy of LN station No. 14. The extent of circumferential dissection of peri-SMA nerve plexus can be adjusted according to the primary disease. However, there is limited evidence on whether adding different extents of peri-SMA nerve plexus dissection to PPMC $(\mathrm{PPMC}+\mathrm{N})$ would be beneficial, ${ }^{2}$ because tumor infiltration into nerve plexus is known to be associated with very poor prognosis. $^{32}$

The goals of PPMC are 1) to approach the peri-SMA

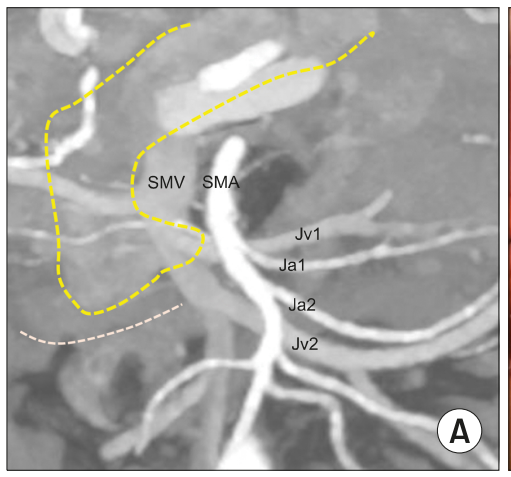

(2)

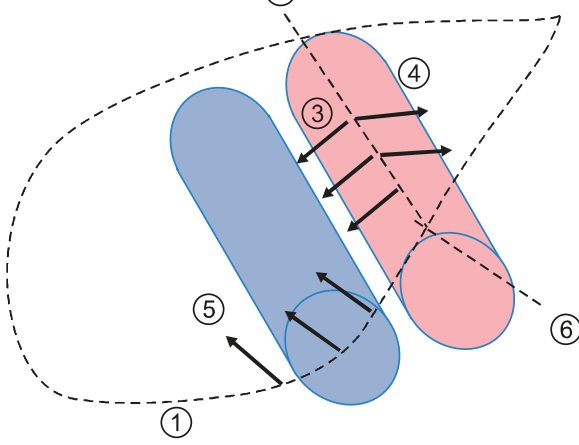

(6)

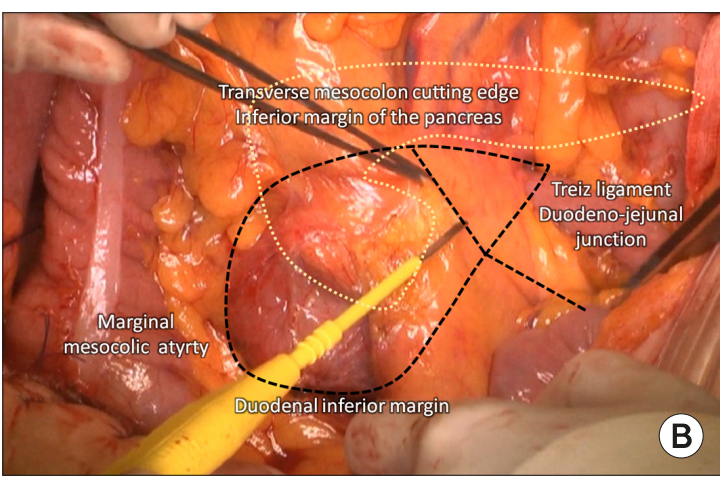

(2)

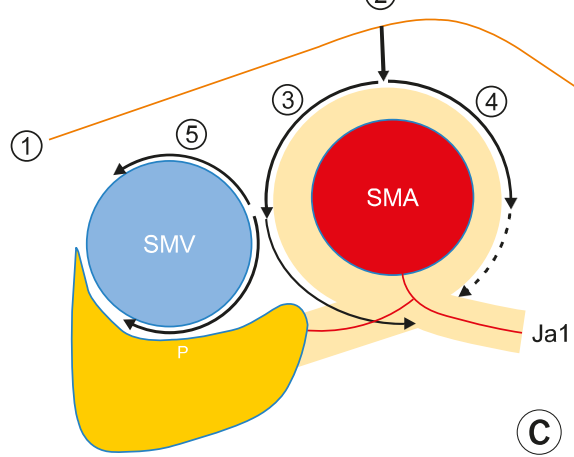

(C)

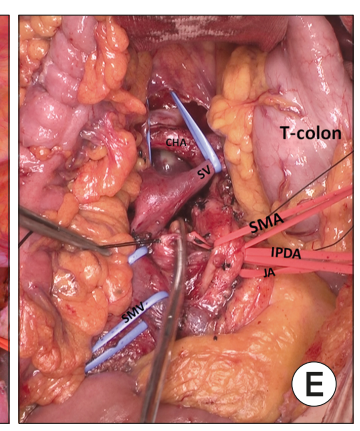

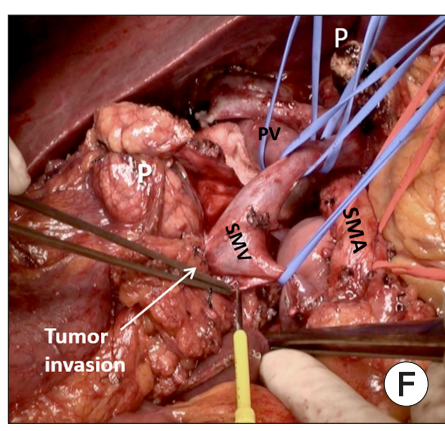

Fig. 2. Operative procedures for PPMC. (A) Identification of anatomy and variations of jejunal arteries and veins. (B) Incision on the root of mesentery and transverse mesocolon. (C) Diagram for dissection at both sides of SMA. (D) Approaching window after cutting MCA. (E) Division of IPDA and separation of SMV from SMA. (F) En bloc removal of specimen. 
area and identify proximal mesenteric vessels to determine resectability, 2) to remove all the soft tissue including LNs around SMV and SMA (14p, 14d, both on the right and left side of the SMA), 3) to dissect SMA nerve plexus in a tailored extent ranging from preservation to whole circumferential dissection, and 4) to remove the whole specimen en bloc, which is critical in oncologic surgery. With PPMC, all soft tissue between the head of the pancreas and mesenteric vessels can be removed en bloc, including the vessels (IPDA with or without Ja1 or Jv1, if indicated), LNs along SMA (14p, 14d, both on the right and left), nerve plexus (pancreatic head nerve plexus I, II, with or without peri-SMA plexus), and other connective tissue. Therefore, the extent of dissection in PPMC includes meso-pancreas excision and the removal of LNs located in left side of the SMA.

\section{OPERATIVE PROCEDURES AND TECHNICAL TIPS FOR EN BLOC PPMC}

The anatomy of mesenteric vessels should be reviewed in detail preoperatively. The anatomy and variations of IPDA, Ja1, and proximal jejunal veins, including the presence of the jejunal vein trunk, and their route in relation to the SMA (dorsal or ventral to the SMA) should be identified (Fig. 2A). The degree of tumor invasion, proximity to major vessels and duodenal inferior margin, which is considered an inferior limit of vein resection, also need evaluation. According to the tumor-vessel interface, Jv1 or Ja1 can be sacrificed ${ }^{33}$ unless Jv1 is forming jejunal trunk draining territories of more than two jejunal arteries. $^{15}$

Among the different techniques of the SMA-first approach, infra- (mesenteric) or supra-colic approaches are recommended for a more complete PPMC. The authors prefer the mesenteric approach proposed by Nakao and Takagi. ${ }^{8}$ The procedure starts with making an incision on the root of the mesentery and transverse mesocolon while pulling the transverse colon upward. The landmarks are the transverse mesocolon at the inferior margin of the pancreas, Treitz ligament, duodenal inferior margin, and the marginal mesocolic artery (Fig. 2B). Through the incision, the SMV and SMA are identified between the mesentery and mesocolon. The SMA is further exposed by making an incision along it. The dissection of tissue on both sides of the SMA starts from the median plane of SMA (Fig. 2C). Omental tissue and some parts of the mesocolon which cover the anterior surface of the pancreatic head can be removed, and mid-colic vessels can be divided for better exposure and a more comfortable dissection without causing any vascular compromise to the transverse colon (Fig. 2D). Once resectability is confirmed, the left side of the SMA is dissected downwards until the level of Ja1 and Ja2, if possible, is identified. It is recommended to identify the IPDA on the left side of the SMA with the most left part of the uncinate process. The IPDA with or without Ja1 is ligated and divided according to anatomical variation and tumor location. Then, the SMV is separated from the SMA, and Jv1 can be divided if necessary (Fig. 2E). Nerve plexuses are dissected according to the determined extent. The splenic vein can be either preserved or divided. The further mobilization of the remnant pancreas with the splenic vein may be helpful in dissecting right side of the SMA and celiac artery. Vessel loops can be used to obtain a better surgical field. Finally, the whole specimen is on the left side of the operator and the specimen is removed en bloc by dividing the SMV as the final step (Fig. 2F).

\section{CONCLUSION}

En bloc PPMC which incorporates the concept of the SMA-first approach, especially the mesenteric approach, can achieve the completeness and efficiency of PD as an oncologic surgery for loco-regional control of pancreatic head cancer. Although it may be barely possible to establish high-level evidence on the benefits of PPMC in improving the local control of pancreatic head cancer, a standard surgical approach should sometimes be challenged by an innovative concept based on theory and experience. PPMC including the removal of all LNs around proximal SMA may be considered as a standard extent of PD for pancreatic head cancer.

\section{CONFLICT OF INTEREST}

The authors have no conflicts of interest or financial ties to disclose. 


\section{ORCID}

Mee Joo Kang: https://orcid.org/0000-0002-9289-3808

Sun-Whe Kim: https://orcid.org/0000-0001-6315-6019

\section{AUTHOR CONTRIBUTIONS}

Conceptualization: SWK. Data curation: MJK, SWK. Formal analysis: MJK, SWK. Methodology: MJK. Project administration: MJK. Visualization: MJK, SWK. Writing original draft: MJK, SWK. Writing - review \& editing: MJK, SWK.

\section{REFERENCES}

1. Nakamura M, Nakashima H, Tsutsumi K, Matsumoto H, Muta $\mathrm{Y}$, Ueno D, et al. First jejunal vein oriented mesenteric excision for pancreatoduodenectomy. J Gastroenterol 2013;48:989-995.

2. Inoue Y, Saiura A, Oba A, Kawakatsu S, Ono Y, Sato T, et al. Optimal extent of superior mesenteric artery dissection during pancreaticoduodenectomy for pancreatic cancer: balancing surgical and oncological safety. J Gastrointest Surg 2019;23:1373-1383.

3. Jiang X, Yu Z, Ma Z, Deng H, Ren W, Shi W, et al. Superior mesenteric artery first approach can improve the clinical outcomes of pancreaticoduodenectomy: a meta-analysis. Int J Surg 2020;73:14-24.

4. Aimoto T, Mizutani S, Kawano Y, Matsushita A, Yamashita N, Suzuki H, et al. Left posterior approach pancreaticoduodenectomy with total mesopancreas excision and circumferential lymphadenectomy around the superior mesenteric artery for pancreatic head carcinoma. J Nippon Med Sch 2013;80:438-445.

5. Kurosaki I, Minagawa M, Takano K, Takizawa K, Hatakeyama K. Left posterior approach to the superior mesenteric vascular pedicle in pancreaticoduodenectomy for cancer of the pancreatic head. JOP 2011;12:220-229.

6. Pessaux P, Rosso E, Panaro F, Marzano E, Oussoultzoglou E, Bachellier $\mathrm{P}$, et al. Preliminary experience with the hanging maneuver for pancreaticoduodenectomy. Eur J Surg Oncol 2009;35: 1006-1010.

7. Weitz J, Rahbari N, Koch M, Büchler MW. The "artery first" approach for resection of pancreatic head cancer. J Am Coll Surg 2010;210:e1-e4.

8. Nakao A, Takagi H. Isolated pancreatectomy for pancreatic head carcinoma using catheter bypass of the portal vein. Hepatogastroenterology 1993;40:426-429.

9. Gockel I, Domeyer M, Wolloscheck T, Konerding MA, Junginger $\mathrm{T}$. Resection of the mesopancreas (RMP): a new surgical classification of a known anatomical space. World J Surg Oncol 2007; $5: 44$.

10. Kawabata Y, Tanaka T, Nishi T, Monma H, Yano S, Tajima Y. Appraisal of a total meso-pancreatoduodenum excision with pancreaticoduodenectomy for pancreatic head carcinoma. Eur J Surg Oncol 2012;38:574-579.

11. Adham M, Singhirunnusorn J. Surgical technique and results of total mesopancreas excision (TMpE) in pancreatic tumors. Eur J Surg Oncol 2012;38:340-345.

12. Hirono S, Kawai M, Okada KI, Miyazawa M, Shimizu A,
Kitahata Y, et al. Mesenteric approach during pancreaticoduodenectomy for pancreatic ductal adenocarcinoma. Ann Gastroenterol Surg 2017;1:208-218.

13. Peparini N, Chirletti P. Mesopancreas: a boundless structure, namely $\mathrm{R} 1$ risk in pancreaticoduodenectomy for pancreatic head carcinoma. Eur J Surg Oncol 2013;39:1303-1308.

14. Gaedcke J, Gunawan B, Grade M, Szöke R, Liersch T, Becker $\mathrm{H}$, et al. The mesopancreas is the primary site for $\mathrm{R} 1$ resection in pancreatic head cancer: relevance for clinical trials. Langenbecks Arch Surg 2010;395:451-458.

15. Ishikawa Y, Ban D, Matsumura S, Mitsunori Y, Ochiai T, Kudo A, et al. Surgical pitfalls of jejunal vein anatomy in pancreaticoduodenectomy. J Hepatobiliary Pancreat Sci 2017;24:394-400.

16. Hosokawa Y, Nagakawa Y, Sahara Y, Takishita C, Nakajima T, Hijikata Y, et al. Surgical outcomes of pancreaticoduodenectomy for pancreatic cancer with proximal dorsal jejunal vein involvement. J Gastrointest Surg 2018;22:1179-1185.

17. Kim JR, Kim H, Kwon W, Jang JY, Kim SW. Pattern of local recurrence after curative resection in pancreatic ductal adenocarcinoma according to the initial location of the tumor. J Hepatobiliary Pancreat Sci 2020. doi: 10.1002/jhbp.854. [in press]

18. Pessaux P, Varma D, Arnaud JP. Pancreaticoduodenectomy: superior mesenteric artery first approach. J Gastrointest Surg 2006; 10:607-611.

19. Shukla PJ, Barreto G, Pandey D, Kanitkar G, Nadkarni MS, Neve R, et al. Modification in the technique of pancreaticoduodenectomy: supracolic division of jejunum to facilitate uncinate process dissection. Hepatogastroenterology 2007;54:1728-1730.

20. Hackert T, Werner J, Weitz J, Schmidt J, Büchler MW. Uncinate process first--a novel approach for pancreatic head resection. Langenbecks Arch Surg 2010;395:1161-1164.

21. Hirota M, Kanemitsu K, Takamori H, Chikamoto A, Tanaka H, Sugita $\mathrm{H}$, et al. Pancreatoduodenectomy using a no-touch isolation technique. Am J Surg 2010;199:e65-e68.

22. Sanjay P, Takaori K, Govil S, Shrikhande SV, Windsor JA. 'Artery-first' approaches to pancreatoduodenectomy. Br J Surg 2012;99:1027-1035.

23. Westgaard A, Tafjord S, Farstad IN, Cvancarova M, Eide TJ, Mathisen $\mathrm{O}$, et al. Resectable adenocarcinomas in the pancreatic head: the retroperitoneal resection margin is an independent prognostic factor. BMC Cancer 2008;8:5.

24. Zhu J, Han D, Li X, Pan F, Ma J, Kou J, et al. Inferior infracolic 'superior mesenteric artery first' approach with a no-touch isolation surgical technique in patients with a borderline resectable cancer of the pancreatic head. Ann Surg Oncol 2016;23(Suppl 5):976-980.

25. Inoue $\mathrm{Y}$, Saiura A, Yoshioka R, Ono Y, Takahashi M, Arita J, et al. Pancreatoduodenectomy with systematic mesopancreas dissection using a supracolic anterior artery-first approach. Ann Surg 2015;262:1092-1101.

26. Sabater L, Cugat E, Serrablo A, Suarez-Artacho G, DiezValladares L, Santoyo-Santoyo J, et al. Does the artery-first approach improve the rate of $\mathrm{R} 0$ resection in pancreatoduodenectomy?: a multicenter, randomized, controlled trial. Ann Surg 2019; 270:738-746.

27. Ramia JM, De-la-Plaza R, Manuel-Vazquez A, Lopez-Marcano A, Morales R. Systematic review of the mesopancreas: concept and clinical implications. Clin Transl Oncol 2018;20:1385-1391.

28. Kawabata Y, Tanaka T, Ishikawa N, Hayashi H, Tajima Y. Modified total meso-pancreatoduodenum excision with pancreaticoduodenectomy as a mesopancreatic plane surgery in borderline resectable pancreatic cancer. Eur J Surg Oncol 2016;42:698705.

29. Japan Pancreas Society. Classification of pancreatic carcinoma. 
4th ed. Tokyo: Kanehara \& Co., Ltd., 2017.

30. Yeo CJ, Cameron JL, Lillemoe KD, Sohn TA, Campbell KA, Sauter PK, et al. Pancreaticoduodenectomy with or without distal gastrectomy and extended retroperitoneal lymphadenectomy for periampullary adenocarcinoma, part 2: randomized controlled trial evaluating survival, morbidity, and mortality. Ann Surg 2002;236:355-366; discussion 366-368.

31. Farnell MB, Pearson RK, Sarr MG, DiMagno EP, Burgart LJ, Dahl TR, et al. A prospective randomized trial comparing standard pancreatoduodenectomy with pancreatoduodenectomy with extended lymphadenectomy in resectable pancreatic head adenocarcinoma. Surgery 2005;138:618-628; discussion 628-630.

32. Kayahara M, Nagakawa $T$, Ueno $K$, Ohta $T$, Tsukioka $Y$, Miyazaki I. Surgical strategy for carcinoma of the pancreas head area based on clinicopathologic analysis of nodal involvement and plexus invasion. Surgery 1995;117:616-623.

33. Katz MH, Fleming JB, Pisters PW, Lee JE, Evans DB. Anatomy of the superior mesenteric vein with special reference to the surgical management of first-order branch involvement at pancreaticoduodenectomy. Ann Surg 2008;248:1098-1102. 